\title{
Social Robotics and Engineering Students: Do They Match? Does Culture Matter?
}

\author{
Mustafa Suphi Erden ${ }^{1} \mathbb{D}$
}

Accepted: 3 December 2019 / Published online: 12 December 2019

(c) The Author(s) 2019

\begin{abstract}
This study investigates the level of familiarity and interest of students towards social robotics through a survey conducted with the Electrical Engineering and Computer Science Students at Heriot-Watt University in the UK and Electrical Engineering Students at Xidian University in China. The results indicate that whereas there is no significant difference in the level of familiarity within the three groups of students and no significant difference in the level of interest between the Electrical Engineering and Computer Science Students at Heriot Watt University, there is a statistically significant difference in the level of interest towards social robotics between the Heriot-Watt University and Xidian University Students. Xidian University Students demonstrate a higher level of interest towards social robotics. The qualitative analysis shows that many of the Xidian University Students are willing to perceive and have robots as companions whereas none of the Heriot-Watt University Students show such or similar tendency. This observation indicates that cultural background plays a significant role in interests and preferences of the students towards social robotics.
\end{abstract}

Keywords Social robotics $\cdot$ Education $\cdot$ Engineering $\cdot$ Culture $\cdot$ Cultural background

\section{Introduction}

This inquiry investigates whether social robotics is an attractive learning and research domain for Electrical Engineering (EE) and Computer Science (CS) Students at Heriot-Watt University (HWU) in the UK and Electrical Engineering Students at Xidian University (XDU) in China? Social robotics is an emergent research domain that brings together engineering and social science researchers and requires knowledge of these two domains. In the core of social robotics lies the question of how we can make the robots more interactive with humans by equipping them with technologies to perceive, interpret, and reason about human activities and at the same time react to these human activities in ways that are similar to and/or acceptable by humans $[8,11]$. In other words, social robotics studies the so-called $3 \mathrm{C}$ tasks, "tasks that require human-robot communication, coordination, or collaboration" [20].

Mustafa Suphi Erden

m.s.erden@hw.ac.uk

1 Electrical, Electronic and Computer Engineering, Institute of Signals, Sensors and Systems, Heriot-Watt University, Edinburgh, UK
Due to the rising interest in research, scholars have started thinking about including social robotics also in education: "Physical robotics has been very popular and effective in... education..., but social robotics in education is almost completely unexplored" [20]. Mead and Mataric [20] investigated using social robotics in middle school classrooms to address the needs of students undergoing changes at the stage of biological development. Edwards et al. [6] tested a social robot for delivery of lessons in a college classroom. Bolea et al. [3] shared the idea that "the bridge from research to teaching is not clear in social robotics". They performed a study towards integrating social robotics with a humanoid robot setup into a taught laboratory in the Industrial Engineering Degree. Ruiz-del-Solar [22] used social robots as keynote speakers in the outreach activities towards students in order to attract them to the electrical, electronic, and computer engineering degrees.

Cultural background is an important factor affecting how people perceive robots, especially considering the acceptability of robots in social life [16] and considering how people interact with them [9]. Lee and Sabanovic [17] conducted a survey in South Korea, Turkey, and the United States in order to investigate the cultural dependencies of preferences for the design and use of robots in daily life. Their study 
emphasized "the importance of performing cross-cultural studies of robots that...delve into...contextually and socially situated dynamics of sense making and interaction" [17]. $\mathrm{Li}$ et al. [19] demonstrated that cultural differences exist in people's perception of likeability, engagement, trust, and satisfaction in human-robot interaction. Rehm suggested that cultural differences were present even in the development of robotic systems "unconsciously influencing how robots look, what we envision with them to do, and how they are programmed to interact with the user" and demonstrated that culture influences how people design affective body movements of a small humanoid robot [21]. Similarly, Shaw-Garlock [24] presented an in-depth discussion of the differences in the seminal work of Cynthia Breazeal from North America and Hiroshi Ishiguro from Japan on humanoid social robots. Haring et al. [14] observed differences in perception of different types of robots by Japanese and Australian university students. In a review paper on social robotics, Dunstan et al. [5] highlighted "cultural robotics" to stress the role of social robots as participant and also creators of culture.

As a roboticist at HWU, I have been particularly interested in how much our students at the engineering departments are familiar with and interested in social robotics. The answer to this question is not trivial because social robotics is highly interdisciplinary in a somewhat uncommon way that the interdisciplinarity is not between two technical domains but between two different research paradigms, engineering and social science. Breazeal et al. [2] stated that social robotics "requires a multidisciplinary approach where the design of social robot technologies and methodologies are informed by robotics, artificial intelligence, psychology, neuroscience, human factors, design, anthropology, and more". Eyssel [10] stated that social roboticists' "interest in human psychology is driven by the motivation to identify principles of human-human interaction in order to extract and model these onto a given platform to mirror psychological processes in a technical system." In the context of social robotics, she added that "Ph.D. candidates in engineering and related fields, ..., are commonly required to provide an empirical "evaluation" study along with the platform they have engineered, designed or refined. ...At the same time, it appears that Ph.D. candidates... may lack the academic training in research methods, experimental design, and statistics" [10]. In that respect social robotics requires not only engineering knowledge, but also knowledge and skills of field studies, questionnaires, statistical analyses, and qualitative and interpretive analyses. Therefore, the familiarity and interest of engineering students towards social robotics is linked to their familiarity with and interest in these broad range of studies. In this survey, I aim at investigating the familiarity with and interest in social robotics through questions not only focusing directly on social robotics per se but also with questions on psychology, user studies, questionnaires, and statistics.

The joint undergraduate program on Telecommunications Engineering between HWU and XDU provides a rare opportunity to study the impact of cultural background of engineering students, in contrast to the impact of educational background. This is because Electrical Engineering students at HWU and the joint Telecommunications Engineering program students at XDU follow exactly the same curriculum in their first 3 years: the content and style (lecture slides) of the courses, the coursework, and the exams are the same. Moreover, one-third of the courses of the joint Telecommunications Engineering program at XDU are delivered by the staff of HWU. These two group of students constitute a pool where the impact of difference in university education is largely eliminated and any observed difference could be more easily attributed to the cultural background. On the other hand, the Electrical Engineering and Computer Science Students in HWU constitute a pool of subjects where the cultural background can be assumed to be similar whereas the educational background differs. A pool of second year students in the two disciplines of HWU and in the joint program of XDU with HWU allow me to study isolated impacts of cultural background and educational background on the question of engineering students' familiarity with and interest in social robotics. To my knowledge, there is no literature focusing specifically on this question, particularly through a controlled comparison where educational and cultural background are largely isolated from each other. Therefore, the results of this study is important to provide evidence on the impact of cultural background in perception of engineering students about social robotics and showing how they differ across the two cultures studied.

\section{Motivation and Hypotheses}

This study was motivated by two reasons that pertain to our specific circumstances at HWU. First, we as the academics in robotics at HWU are increasingly including social robotics topics in our research and therefore we would like to know whether this is appealing to our students in their undergraduate projects and post-graduate studies, both in Computer Science and Electrical Engineering. Besides that, traditionally social robotics is associated with Computer Science rather than Electrical Engineering and therefore there is more social robotics activity going on in the Computer Science department than in the Electrical Engineering department at HWU. We are curious whether social robotics is as familiar and interesting to the Electrical Engineering Students as to the Computer Science Students. This comparison has been motivated by my observation that different 
educational programs might result in different world-views with students [7]. Accordingly I hypothesised that:

Hypothesis 1a There is a difference in the level of familiarity with social robotics between Computer Science students and Electrical Engineering students at Heriot-Watt University.

Hypothesis $\mathbf{1 b}$ There is a difference in the level of interest in social robotics between Computer Science students and Electrical Engineering students at Heriot-Watt University.

Second, at HWU, we have a joint engineering program with the XDU in Xi' an, China. Starting from 2018, each year a cohort of electrical engineering students from XDU have been taught in HWU in Edinburgh in their fourth years. They are conducting student projects some of which are part of the running research projects and they will potentially like to pursue post-graduate studies at HWU. For these purposes, we would like to know the level of interest and familiarity of our Chinese students towards social robotics in comparison to our local students and would like to develop and propose projects according to their interests. The results of this survey will inform us about whether our students are familiar with and interested in social robotics and whether there is any difference in the level of interest between the students from Edinburgh and Xi' an. Accordingly I hypothesised that:

Hypothesis 2a There is a difference in the level of familiarity with social robotics between Heriot-Watt University students and Xidian University students.

Hypothesis $\mathbf{2 b}$ There is a difference in the level of interest in social robotics between Heriot-Watt University students and Xidian University students.

I believe that the results of this study are interesting also for the social robotics community at large. The results related to the impact of cultural background in perception of social robotics might be of interest to even a larger community including social scientists who focus on ethnicity and life-style factors in perception of technology, and to market analysts of industry commercializing interactive robotic products.

\section{Methodology and Methods}

In this study, I aim at understanding the students' level of familiarity with and interest in social robotics. The study does not aim at measuring their actual level of knowledge of social robotics concepts; rather the interest here is to understand the level of their general familiarity with and interest in social robotics based on what they have heard and got to know from their surroundings and regardless of their having learned of any technical details. The survey was conducted on students with the same level of technical education in the two universities. This approach makes the assumption that the social and cultural environment is effective in order to shape the motivation and interest of students towards an emergent field like social robotics, and this environment makes a difference in that respect regardless of the students having previously acquired the same level and similar education. The familiarity and interest that result from the environmental impacts can be captured by simply asking the students whether they would agree or not agree with the related statements and letting them express themselves in relation to those.

Different societal causes might impact students to have high or low interest in an emergent domain of study. Lee and Sabanovic [17] investigated the impact of religion and media exposure on the preferences towards social robots (though did not reach conclusive results) and they referenced to the reports $[15,25]$ that suggest that the active efforts of the government, industry, and academia have been influential on social acceptance of robotics in Japan. Walden et al. [26] reported that the mental model of seniors about robots were "shaped by their recent experiences with advanced communications technologies and mediated representations of robots in popular culture". Destephe et al. [4] demonstrated with a survey that the attitude of the participants towards robots in general affected their perception of the specific humanoid robot they experimented with: "Participants who had positive views toward robots rated our robot and its motions less eerie and more attractive than those with negative views". Bartneck et al. [1] conducted a cross-cultural survey in seven countries which suggested that people from different cultures had different attitudes towards robots. Lee et al. [18] identified variable cultural models of robotic technology and variable cultural meaning of home context, which shaped the different expectations and preferences of participants of different culture from future domestic robots.

In the current study I aim at showing that the cultural background-which I consider as a compound of all the societal causes surrounding the individual in their dailylife- has an impact in shaping the preferences of undergraduate students. My assumption is that as a result of the impact of such various societal causes, ontologically [12], a cultural background exists and surrounds the students to shape their preferences in choosing the study matters. Epistemologically [12], we can measure the impact of this cultural background by investigating the level of interest in and familiarity with a specific topic. 


\subsection{Questionnaire}

A paper based survey was conducted, composed of 28 closed-form questions, where the students marked their level of agreement with the statements according to five level Likert-scale, and 6 open-ended questions. The open-ended questions allowed the students to express their ideas in written form and aimed to capture anything that was perceived by them as important but was not covered in the closed form questions. I believe to have addressed with these open-ended questions "the need to allow people to speak in their own terms ... to understand their distinctive perspectives" [13]. Among the closed form questions, seven questions aimed at measuring the level of familiarity of the students with social robotics, seven questions aimed at measuring their level of interest in social robotics, three questions aimed at identifying for what purpose they would like to use robotics knowledge, seven questions aimed at understanding their view on to what and for what social robotics knowledge might be related and useful, and three questions asked about different activities students might like to do with robots. Half of the closed form questions allowed me to compute indexes for familiarity and interest of each participant. These indexes were used to compare the groups with each other. The other half of the closed form questions and responses to the openended questions allowed me to investigate their motivations underlying the differences across the groups.

The motivation in formulating the survey questions was to investigate whether the research aspects that are commonly applied in social robotics but that are not necessarily such common in other fields of robotics-specifically, user studies, cross cultural studies, surveys, statistical analyses, and concepts of psychology - were familiar and of interest to the students. The goal of this study was specifically to focus on social robotics and not to get confused with other robotics fields. Obviously, all robotics knowledge and research techniques, ranging from control to mechatronics design and to surveys, also apply to social robotics as to the other robotics domains. In this study I wanted the students to clearly distinguish social robotics from other fields; therefore, I focused on the aspects that were more common to social robotics than to other robotics fields.

The study was approved by the Ethics Committee of the School of Engineering and Physical Sciences at HWU. All participants received and signed an Informed-Consent Form prior to the survey.

\subsection{Participants}

The survey was conducted with three groups of second year university students:
1. HWU Electrical Engineering Students (HW-EE): 26 students.

2. HWU Computer Science Students (HW-CS): 19 students.

3. XDU Electrical Engineering Students (XD-EE): 46 students.

\subsection{Questions}

Among the closed form questions, where the students marked the level of their agreement with the statements, the following seven questions aimed at measuring the level of familiarity of the students with social robotics, as they had familiarity connotation with the verbs and phrases such as "I heard about", "I know what", "is related to", "is used in" and pointed to a relevant or correct information:

(F1) I heard about social robotics.

(F2) I know what social robotics is.

(F3) Social robotics is related to psychology.

(F4) Statistics is used in social robotics studies.

(F5) I know what usability of a robot means.

(F6) I know what acceptability of a robot means.

(F7) I know what statistically significant result means.

The following seven questions aimed at measuring the level of interest of the students in social robotics, as these questions indicated an interest with the verbs and phrases such as "I want to", "I am interested", "I like":

(I1) I want to learn about social-robotics.

(I2) I am interested in doing research in social-robotics.

(I3) I like reading on psychology.

(I4) I am interested in interacting with people for testing systems.

(I5) I am interested in preparing questionnaires.

(I6) I am interested in conducting questionnaire studies with people.

(I7) I like statistics and conducting statistical tests.

The following four questions aimed at identifying for what purpose the students would like to use robotics knowledge, as they all involved the phrase "to develop robots that/ for":

(P1) I like to develop robots that understand humans' emotions.

(P2) I like to develop robots that convey emotions to humans (happy robot, sad robot, etc.).

(P3) I am interested in developing robots for daily life and home usage.

(P4) I am interested in developing robots for factories. 
Among the remaining closed form questions seven of them (R1-R7) indicated what social robotics knowledge might be related to or useful for (such as for engineering, for neuroscience, to make robots better understand humans, etc.) and three of them (A1-A3) indicated different activities students might like to do with robots (designing, building, programming).

The following six open-ended questions allowed the students to express their ideas in written form in order to allow capturing anything that was perceived by them as important but was not covered in the closed form questions.

(O1) Please describe what you think Social Robotics is:

(O2) Please describe how and for what purpose Social Robotics Knowledge could be useful:

(O3) Please name any techniques you know/imagine that could be used in Social Robotics:

(O4) Please state why you would or you would not be interested in Social Robotics:

(O5) Please state what you would like doing with robots:

(O6) Please state what you would like to use robots for:

\subsection{Analysis}

The answers to the closed form questions were analysed using the SPSS tools for computing the means of the responses for each group, performing One-Way ANOVA tests to find any significant difference in the responses of the three groups, and performing post hoc tests with both Least Significant Difference (LSD) Test and Independent Samples $T$ Test to mutually compare the responses of the three groups. I used the statistical significance level of $p=0.05$ for all statistical tests throughout the paper.

A familiarity index, $F$-In, and an interest index, I-In, were computed for each participant by averaging the responses to the familiarity (F1-F7) and interest (I1-I7) related questions, respectively. A reliability analysis of Cronbach's alpha was carried out separately on the familiarity index and interest index comprising the corresponding seven questions, respectively. Cronbach's alpha showed both of the indexes to reach acceptable reliability, with $\alpha=0.703$ for $F$-In and 0.857 for $I-\operatorname{In}(\alpha>0.7$ is required for high internal consistency). All questions in both indexes appeared to be worthy of retention, resulting in a decrease in the alpha of the corresponding index if deleted.

The mean $F$-In and I-In of each subject group was computed and compared with each other. In order to verify that parametric tests (ANOVA and $T$ tests) could be applied on the data set, I ran Kolmogorov-Smirnov D test to check the normality of each of the six data-groups ( $F$-In and I-In for each of the three subject groups) and Levene's Test of Equality of Error Variances Based on Mean to check the homogeneity of variance within each of the three compared data-groups for the two indices ( $F$-In of the three subject groups and $I-I n$ of the three subject groups). All six datagroups passed the normality test (the null hypothesis of no difference between the observed data distribution and a normal distribution was not rejected as $p$ values were larger than 0.05: 0.072 for I-In-XD and larger than 0.200 for the rest) and the three data-groups for each of $F-I n$ and $I-I n$ passed the homogeneity of variance test (the null hypothesis of error variance of the three data-groups is equal was not rejected as $p$ values were larger than 0.05: 0.179 and 0.065, for $F$-In and I-In, respectively).

Afterwards, non-parametric Independent Samples Mann-Whitney U test was applied to compare the mean of each of the 28 closed form questions across the HWU (combined HW-EE and HW-CS) and XDU (XD-EE) students, in order to find out which of the individual questions made a difference across the students due to their cultural background.

Finally, I conducted a thematic analysis [23] over the responses to the open-ended questions. I followed the method of searching for repetitions as pointed out by Ryan and Bernard [23]: "Some of the most obvious themes in a corpus of data are topics that occur and reoccur or are recurring regularities. The more the same concept occurs in a text, the more likely it is a theme". Rather than looking for repetitions in a single text, I looked for repetitions that occur across the responses of the participants of a group but not of another group.

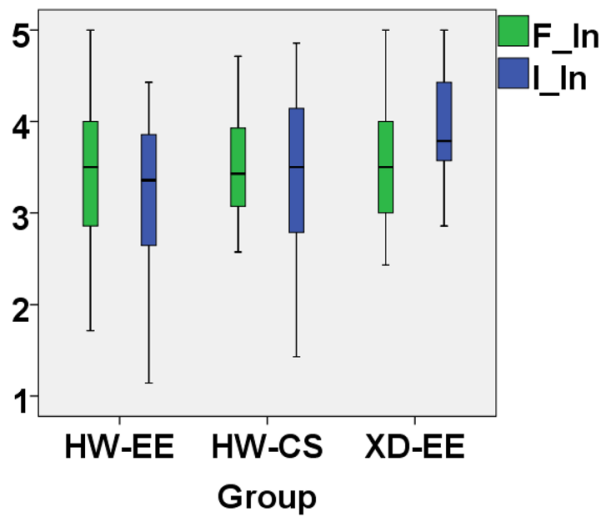

Fig. 1 Mean values of Familiarity and Interest Indexes (F-In, I-In) for the three groups of (1) HWU Electrical Engineering Students (HWEE), (2) HWU Computer Science Students (HW-CS), and (3) Xidian University Electrical Engineering Student (XD-EE) 


\section{Findings}

\subsection{Quantitative Analysis}

\subsubsection{Mean Values of Familiarity and Interest Indexes for the Three Groups}

In Fig. 1 we see the mean values of Familiarity and Interest Indexes for the three groups of (i) HWU Electrical Engineering Students (HW-EE), (ii) HWU Computer Science Students (HW-CS), and (iii) XDU Electrical Engineering Students (XD-EE). We observe that the mean values of the Familiarity Index ( $F$-In_HW-EE: $3.46 ; F$-In_HW-CS: 3.52 ; F-In_XD-EE: 3.50$)$ and Interest Index (I-In_HW-EE: 3.26; I-In_HW-CS: 3.39; I-In_XD-EE: 3.91) are above the neutral value 3 for all three groups. Therefore, we can conclude that all three groups have some familiarity with and interest in social robotics.

\subsubsection{Comparison of Groups for Familiarity and Interest}

One-Way ANOVA applied to the $F$-In of the three groups (HW-EE, HW-CS, and XD-EE) did not find a significant effect of group on the $F$-In measures [degrees of freedom $(d f)=2$; mean square $(m s)=0.033 ; F$ statistics $(F)=0.072$; significance $(p)=0.931]$. This analysis rejected the hypothesis that there is any significant difference between the familiarities of the three groups with social robotics. Then, I combined the HW-EE and HW-CS students under a single group as HWU Students and compared their $F$-In with those of the XD-EE students. The mean of $F$-In for the combined HWU Students was 3.48 against the mean of the XDU Students of 3.50, and a $T$ test run on these two groups found no significant difference between the $F$-In means of HWU and XDU Students, with a significance level $p=0.766$. All these concluded that neither the department nor the university made any significant difference in the familiarity of the students. Hence both Hypothesis 1(a) and Hypothesis 2(a) were rejected.

One-Way ANOVA applied to the I-In of the three groups (HW-EE, HW-CS, and XD-EE), on the other hand, found a significant effect of group on the $I$-In measures $(d f=2$; $m s=4.449 ; F=7.858 ; p=0.001)$. The post hoc analysis of multiple comparison with LSD found significant difference between the means of HW-EE and XD-EE $(p<0.001)$ and also between the means of HW-CS and XD-EE $(p=0.023)$; but did not find any significant difference between the means of HW-EE and HW-CS ( $p=0.377)$. After combining the HW-EE and HW-CS students, the mean of I-In for the HWU Students was 3.31 against the mean for the XDU Students of 3.91. The $T$ test found a statistically significant difference between the I-In means of HWU and XDU Students with a significance level $p<0.001$. These analyses concluded that (i) there is no difference in the level of interest in social robotics across Electrical Engineering and Computer Science students at HWU, (ii) there is a significant difference in the level of interest in social robotics across the students in HWU (both HW-EE and HW-CS) and the students in XDU (XD-EE). Hence, Hypothesis 1(b) was rejected, but Hypothesis 2(b) was confirmed. The means of the I-In index showed that students at XDU have significantly higher interest in social robotics than the students in HWU.

\subsubsection{Comparison of Responses to Each Question}

In order to have an understanding of which questions made a difference between the students of the two departments at Heriot-Watt University (HW-EE vs. HW-CS) and between the students of HWU and XDU (HW-EE and HW-CS vs. XD-EE), non-parametric Independent Samples Mann-Whitney $U$ Test was run for each question.

Only three questions made a statistically significant difference between the responses of HW-EE and HW-CS students:

I5 ( $p=0.037)$ : HW-CS mean =3.00; HW-EE mean $=2.15$.

R2 $(p=0.009)$ : HW-CS mean $=3.74 ;$ HW-EE mean $=4.32$.

A2 $(p=0.016)$ : HW-CS mean $=3.37 ;$ HW-EE mean $=4.15$.

The mean of the answers to question I5 was larger with the Computer Science students (3.00) compared to the Electrical Engineering students (2.15) at HWU. This means that Computer Science students showed more interest towards preparing questionnaires and conducting questionnaire studies. This can be related to the fact that social robotics and related questionnaire studies are more common in Computer Science research than in Electrical Engineering Research, especially in HWU. However, we should note that the difference in this single question did not lead to any significant difference in the Interest Index of the two groups.

The mean of the answers to questions R2 (Social Robotics is an engineering domain) and A2 (I like building robots) were larger with the Electrical Engineering students (4.32, 4.15) compared to the Computer Science students (3.74, 3.37) at HWU. This means, as expected, the Electrical Engineering Students preferred the robotics activities that relate to engineering of hardware development, to which they are more accustomed, compared to the Computer Science Students.

The questions which made a statistically significant difference across the HWU (HW-CS and HW-EE combined) and XDU (XD-EE) students were identified as follows: 
F3 $(p=0.015)$ : HWU mean $=3.93 ;$ XDU mean $=4.24$.

F4 $(p=0.018)$ : HWU mean $=3.91 ;$ XDU mean $=4.30$.

F7 $(p<0.001)$ : HWU mean $=4.14$; XDU mean $=3.24$.

I1 $(p=0.016)$ : HWU mean $=3.93$; XDU mean $=4.29$.

I2 $(p=0.020)$ : HWU mean $=3.45$; XDU mean $=4.02$.

I3 $(p=0.001)$ : HWU mean $=3.16$; XDU mean $=4.09$.

I5 $(p<0.001)$ : HWU mean $=2.51 ;$ XDU mean $=3.76$.

I6 $(p=0.010)$ : HWU mean $=2.94$; XDU mean $=3.64$.

$\mathrm{P} 1(p=0.002)$ : HWU mean $=3.66 ; \mathrm{XDU}$ mean $=4.27$.

$\mathrm{P} 2(p=0.002)$ : HWU mean $=3.67$; XDU mean $=4.27$.

$\mathrm{R} 5(p=0.030)$ : HWU mean $=3.93 ; \mathrm{XDU}$ mean $=4.24$.

The means of the answers to questions F3-F4 were larger with the XDU students $(4.24 ; 4.30)$ compared to the HWU students $(3.93 ; 3.91)$. This means that XDU students were more familiar with the fact that psychology and statistics are related to social robotics. On the other hand, the mean of the answers to question F7 was larger with the HWU students (4.14) compared to the XDU students (3.24) suggesting that XDU students were less familiar with the concept of statistical significance. We should note that the differences in the answers to these three questions did not lead to any significant difference in the Familiarity Index of the two groups.

The means of the answers to questions I1-I3, I5-I6 and P1-P2 were larger with the XDU Students compared to the HWU Students. Among these, the differences related to questions I1-I3, I5-I6 are already reflected in the difference in the Interest Index of the two groups. However, the differences related to questions $\mathrm{P} 1$ and $\mathrm{P} 2$ are remarkable as they reflect that XDU students $(4.27 ; 4.27)$ more strongly agreed than the HWU students $(3.66 ; 3.67)$ that they like to develop robots that understand human's emotions and that convey emotions to humans. This quantitative result strongly resonates with the results of the qualitative analysis below.

Finally, the mean of the answers to question R5 (Socialrobotics knowledge is useful for programming robots) was larger with the XDU students compared to the HWU students; suggesting that XDU students more strongly agreed that social robotics was related to programming rather than building robots (as the means of the answers to a previous question R4 [Social-robotics knowledge is useful for building robots] did not make any difference between HWU and XDU students). Again this difference resonates with the higher level of interest of the XDU students with social robotics.

\section{Qualitative Analysis}

The qualitative analysis was based on the responses of the students to the open-ended questions in the survey. After a first reading of all the responses, I realized that many of the XDU Students used words and phrases that referred to an understanding and willingness of using robots as companions. The HWU students, on the other hand, did not use similar words and phrases; rather, they mostly used words and phrases that referred to an understanding and willingness of using robots as utility equipment, such as using in the factories, for repetitive and dull tasks, for cleaning, and in dangerous situations. These latter were shared also by some of the XDU students, but the interesting point was that none of the HWU students expressed any inclination towards an understanding and wiliness of using robots as companions. After having this first impression, I extracted the phrases used by XDU students given below. 17 of the 46 XDU students expressed at least one or more of these words and phrases in their responses. None of the HWU students expressed any of these or any other words or phrases similar to these.

\footnotetext{
Phrases that were used by 17 of 46 Xidian University students and that or similar of which were not used by any of the Heriot-Watt University students (spelling and grammar errors have been retained as expressed by the students):

Accompany lonely people, helpmate for the elders who live alone, avoid loneliness, care of the lonely old people, babysitting, take care of olds, to help the lonely people feeling warm and homeful, make robots more than robots ("it" becomes "he" or "she"), change the lifestyle, magical, make everyone happier, playing with me, play games, share emotions, chatting, doing fitness, sharing things which happened today, make friends with it, regard them as a friend, I hope robots can be our friends, talking with me when I am boring, playing tennis or badminton with me, playing sports, electronic pets, a robot with emotion can be our friend, entertainment, care about me, taking care of children, I will not "use" robots.
}

\section{Discussion}

The findings presented here point out to the following conclusions.

1. Electrical Engineering and Computer Science Students at Heriot-Watt University and Electrical Engineering Students at Xidian University have some familiarity with and interest in social robotics.

This observation implies for our local context at HeriotWatt University that we can pursue running social robotics projects and encourage our students to take social robotics topics in their projects and post-graduate studies.

2. There is no significant difference between the Electrical Engineering and Computer Science Students at HeriotWatt University regarding to their level of familiarity and interest towards social robotics. 
This observation is important in order to eliminate the hesitations for introducing social robotics in Electrical Engineering Department in comparison to Computer Science Department at HWU. The results of this survey show that Electrical Engineering Students are as familiar with and interested in social robotics as the Computer Science Students.

3. The students at Xidian University are more interested in social robotics than the students at Heriot-Watt University.

This observation has both local and general implications. The local implication for the Electrical Engineering Department at Heriot-Watt University is that we can perhaps attract more students to social robotics studies from among the Xidian University students when they spend their fourth year of the joint program in the Heriot-Watt University Campus in Edinburgh. This might be an opportunity to increase the number and depth of social robotics research at Heriot-Watt University. The more general implication of this observation is related to the following observation.

4. The students at Xidian University perceive and are willing to use robots as companions whereas the students at Heriot-Watt University do not show any evidence of tendency in this direction and they rather perceive and are willing to use robots as utility equipment.

Both the qualitative analysis presented above and the difference in the quantified responses to the questions P1-P2 support this observation. Therefore, this study provides combined qualitative and quantitative features which "contribute complementary form of evidence in addressing the same issue" [13].

The implication of this observation is that the level of interest of students towards an emergent research field such as social robotics is dependent on the culture that surrounds them. The results show evidence for that the level of interest in the Chinese context in Xi' an is higher towards social robots than in the British-Scottish context in Edinburgh. Most importantly, the way that robots are perceived or the way how they are imagined to take place in the lives of humans seem to differ. Whereas the students at Heriot-Watt University are willing to use the robots only for utilitarian purposes, such as in dull and dangerous tasks, the students at Xidian University are willing to use robots also as companions as if they were like their human friends in their social and daily lives. I think, the term companion is the key here to distinguish the tendency that is observed with the Xidian University students but not with the Heriot-Watt University students.
This observation provides evidence to the more general argument that robots are more easily perceived as companions in some cultural environments compared to some others. This implies that social robotics research, particularly the ones that perform user studies, should take into account the cultural background of the context the research is being conducted. The results obtained in one particular cultural context might not necessarily generalize to another, or a research or technology output that shows success in one cultural context, might not necessarily achieve the same success in another context. This observation also implies that, as argued by Lee and Sabanovic [17], the development and marketing strategies for socially interactive robot technologies should seriously take into account the cultural background of their target end-users.

\section{Conclusion and Recommendations}

This study investigated the level of familiarity and interest of students towards social robotics through a survey conducted with the Electrical Engineering and Computer Science students at Heriot-Watt University and Electrical Engineering students at Xidian University. The results indicated that whereas there is no significant difference in the level of familiarity across the three groups of students and no significant difference in the level of interest between the Electrical Engineering and Computer Science students at Heriot Watt University, there is a statistically significant difference in the level of interest towards social robotics between the Heriot-Watt University and Xidian University students. Xidian University students demonstrate a higher level of interest towards social robotics. The qualitative analysis conducted over the responses of the students revealed that many of the Xidian University students were motivated to perceive and have robots as companions whereas none of the Heriot-Watt University students showed such or similar tendency. This observation strongly supports the claim that cultural background plays a significant role in determining the interests and preferences of students towards an emergent research and study field such as social robotics. This observation also supports that cultural background should be taken into account while conducting research, development, and marketing activities related to robots that interact with people.

\section{Compliance with Ethical Standards}

Conflict of interest The author declares that he has no conflict of interest.

Open Access This article is licensed under a Creative Commons Attribution 4.0 International License, which permits use, sharing, adaptation, distribution and reproduction in any medium or format, as long 
as you give appropriate credit to the original author(s) and the source, provide a link to the Creative Commons licence, and indicate if changes were made. The images or other third party material in this article are included in the article's Creative Commons licence, unless indicated otherwise in a credit line to the material. If material is not included in the article's Creative Commons licence and your intended use is not permitted by statutory regulation or exceeds the permitted use, you will need to obtain permission directly from the copyright holder. To view a copy of this licence, visit http://creativecommons.org/licenses/by/4.0/.

\section{References}

1. Bartneck C, Suzuki T, Kanda T, Nomura T (2007) The influence of people's culture and prior experiences with Aibo on their attitude towards robots. AI Soc 21:217-230

2. Breazeal C, Dautenhahn K, Kanda T (2016) Social Robotics. In: Siciliano B, Khatib O (eds) Springer handbook of robotics, Springer Handbooks. Springer, Cham, pp 1935-1971

3. Bolea Y, Grau A, Sanfeliu A (2016) From research to teaching: integrating social robotics in engineering degrees. Int J Comput Electr Autom Control Inf Eng 10(6):1026-1029

4. Destephe M, Brandao M, Kishi T, Zecca M, Hashimoto K, Takanishi A (2015) Walking in the uncanny valley: importance of the attractiveness on the acceptance of a robot as a working partner. Front Psychol 6, 204. https://doi.org/10.3389/fpsyg.2015.00204

5. Dunstan BJ, Silvera-Tawil D, Koh JTKV, Velonaki M (2016) Cultural robotics: robots as participants and creators of culture. In: International workshop on cultural robotics, CR 2015: cultural robotics, LNCS, vol 9549, pp 3-13

6. Edwards A, Edwards C, Spence PR, Harris C (2016) Robots in the classroom: differences in students' perception of credibility and learning between "teacher a robot" and "robot as teacher". Comput Hum Behav 65:627-634

7. Erden MS (2003) Positivistic tendencies due to engineering education. In: Proceedings of ACE2003-6th IFAC symposium on advances in control education, Oulu, Finland, pp 389-394

8. Erden MS (2013) Emotional postures for the humanoid-robot nao. Int J Social Robot 5:441-456

9. Evers V, Maldonado H, Brodecki T, Hinds P (2008) Relational vs. group self-construal: untangling the role of national culture in HRI. In: Proceedings of ACM/IEEE international conference on human-robot interaction (HRI), March 12-15, Amsterdam, The Netherlands, pp 255-262

10. Eyssel F (2017) An experimental psychological perspective on social robotics. Robot Autonom Syst 87:363-371

11. Ge SS (2007) Social robotics: integrating advances in engineering and computer science. In: Proceedings of the electrical engineering/electronics, computer, telecommunications and information technology international conference. https://pdfs.semanticsc holar.org/cc1f/f40a8277ab86fd0341f287a251fd37b15f9c.pdf. Accessed 21 Dec 2018

12. Grix J (2002) Introducing students to the generic terminology of social research. Politics 22(3):175-186

13. Hammersley M (2013) What is qualitative research. Bloomsbury Academic, London, pp 1-20

14. Haring KS, Silvera-Tawill D, Watanabe K, Velonaki M (2016) The influence of robot appearance and interactive ability in HRI: a cross-cultural study. In: International conference on social robotics, ICSR 2016: social robotics, LNCS, vol 9979, pp 392-401

15. Ito K (2007) Astroboy's birthday: robotics and culture in contemporary Japanese society. Paper presented at EASTS 2007, from http://stspo.ym.edu.tw/easts/2007/Ito.pdf
16. Kaplan F (2004) Who is afraid of the humanoid? Investigating cultural differences in the acceptance of robots. Int J Hum Robots 1(3): $1-16$

17. Lee HR, Sabanovic S (2014) Culturally variable preferences for robot design and use in South Korea, Turkey, and the United States. In: Proceedings of the conference on human robot interaction (HRI), March 3-6, Bielefeld, Germany

18. Lee HR, Sung JY, Sabanovic S, Han J (2012) Cultural design of domestic robots: a study of user expectations in Korea and the United States. In: Proceedings of the IEEE-RAS international symposium on robot and human interactive communication (ROMAN), Sep. 9-13, Paris, France, pp 803-808

19. Li D, Rau PLR, Li Y (2010) A cross-cultural Study: effect of robot appearance and task. Int J Soc Robot 2:175-186

20. Mead R, Mataric MJ (2015) Social robotics in the middle school classroom: increasing student STEM achievement-related choices. In: The 10th ACM/IEEE international conference on human-robot interaction (HRI 2015) education workshop: how to design and teach courses in human-robot interaction, Portland, Oregon, March 2015. http://robotics.usc.edu/ rossmead/docs/2015/2015M eadMataric_HRI-E2015.pdf

21. Rehm M (2012) Experimental design for cross-cultural interactions: a case study on affective body movements for HRI. In: Proceedings of the IEEE-RAS international conference on humanoid robots, Nov. 29-Dec. 1, Osaka, Japan, pp 78-83

22. Ruiz-del-Solar J (2010) Robotics-centered outreach activities: an integrated approach. Electr Eng Trans Educ 53(1):38-45

23. Ryan G, Bernard H (2003) Techniques to identify themes. Field Methods 15(1):85-109

24. Shaw-Garlock G (2009) Looking forward to sociable robots. Int J Soc Robot 1:249-260

25. Wagner C (2009) The Japanese way of robotics: interacting naturally with robots as a national character? In: Proceedings of ROMAN 2009, electrical engineering, pp 510-515

26. Walden J, Jung EH, Sundar S, Johnson AC (2015) Mental models of robots among senor citizens. Interact Stud 16(1):68-88

Publisher's Note Springer Nature remains neutral with regard to jurisdictional claims in published maps and institutional affiliations.

Mustafa Suphi Erden received the B.S., M.S., and Ph.D. degrees in Electrical and Electronics Engineering from Middle East Technical University, Ankara, in 1999, 2001, and 2006. Between 1999 and 2006, he was a Research Assistant in the same department. From 2007 to 2012 he was a postdoctoral researcher, successively in Delft University of Technology, the Netherlands; in Ecole Nationale Supérieure de Techniques Avancées-ParisTech, France; in Univ. Pierre \& Marie Curie - Paris 6, France. In 2012 he received the European Union Marie Curie Intra-European Fellowship with his project "Skill Assistance with Robot for Manual Welding" - SkillAssist. Between 2012 and 2014, he was with Ecole Polytechnique Fédérale de Lausanne, Switzerland, with this fellowship. He is now an Assistant Professor with the School of Engineering and Physical Sciences at Heriot-Watt University in Edinburgh, UK. His research interests include physical human-robot interaction, assistive robotics, skill assistance, mechatronics design, medical robotics, walking robots, machine learning, and social robotics. He has been the PI of research projects funded in total with around $£ 234 \mathrm{~K}$ by the Engineering and Physical Sciences Research Council (EPSRC) of UK and $£ 200 \mathrm{~K}$ by industry. 